\title{
Mathematical sense-making in quantum mechanics: An initial peek
}

\author{
Benjamin W. Dreyfus, ${ }^{1,2, *}$ Andrew Elby, ${ }^{3,4}$ Ayush Gupta, ${ }^{3,5}$ and Erin Ronayne Sohr ${ }^{3}$ \\ ${ }^{1}$ Department of Physics and Astronomy, George Mason University, Fairfax, Virginia 22030, USA \\ ${ }^{2}$ STEM Accelerator Program, George Mason University, Fairfax, Virginia 22030, USA \\ ${ }^{3}$ Department of Physics, University of Maryland, College Park, Maryland 20742, USA \\ ${ }^{4}$ Department of Teaching and Learning, Policy and Leadership, \\ University of Maryland, College Park, Maryland 20742, USA \\ ${ }^{5}$ Keystone Program, A. J. Clark School of Engineering, \\ University of Maryland, College Park, Maryland 20742, USA
}

(Received 24 June 2017; published 28 December 2017)

\begin{abstract}
Mathematical sense-making-looking for coherence between the structure of the mathematical formalism and causal or functional relations in the world-is a core component of physics expertise. Some physics education research studies have explored what mathematical sense-making looks like at the introductory physics level, while some historians and "science studies" have explored how expert physicists engage in it. What is largely missing, with a few exceptions, is theoretical and empirical work at the intermediate level—upper division physics students—especially when they are learning difficult new mathematical formalism. In this paper, we present analysis of a segment of video-recorded discussion between two students grappling with a quantum mechanics question to illustrate what mathematical sensemaking can look like in quantum mechanics. We claim that mathematical sense-making is possible and productive for learning and problem solving in quantum mechanics. Mathematical sense-making in quantum mechanics is continuous in many ways with mathematical sense-making in introductory physics. However, in the context of quantum mechanics, the connections between formalism, intuitive conceptual schema, and the physical world become more compound (nested) and indirect. We illustrate these similarities and differences in part by proposing a new symbolic form, eigenvector eigenvalue, which is composed of multiple primitive symbolic forms.
\end{abstract}

DOI: 10.1103/PhysRevPhysEducRes.13.020141

\section{INTRODUCTION}

Learning quantum mechanics (QM) poses many challenges above and beyond the skills that are developed in introductory physics [1-3]. Some of these challenges are similar to those faced in introductory physics [4], but some are new: students have to learn to activate new ontologies for quantum entities [5-10], navigate multiple interpretations of quantum mechanics [11,12], calculate with new mathematical structures $[13,14]$, and engage with fundamental issues of quantum measurement [15-17].

To this list we add another essential element of quantum mechanics expertise: mathematical sense-making, by which we mean looking for coherence between the mathematical formalism and functional or causal relations in the world $[18,19]$. As a brief example of why mathematical sense-making in QM (and more generally) is not the "simple sum" of conceptual understanding and facility

\footnotetext{
*bdreyfu2@gmu.edu
}

Published by the American Physical Society under the terms of the Creative Commons Attribution 4.0 International license. Further distribution of this work must maintain attribution to the author(s) and the published article's title, journal citation, and DOI. with formalism, consider the incommensurability of pairs of physical quantities corresponding to noncommuting QM operators, such as position and momentum or the $x$ and $y$ components of spin. We can imagine students who develop an adequate conceptual understanding of the indefiniteness of physical quantities in QM and also develop formal problem-solving facility with commutation relations, but who do not "translate" back and forth between the conceptual and formal or mathematical knowledge to "read off" incommensurability from commutation relations, or relate noncommuting operators as represented in position space to properties of Fourier transformations (which transform very narrow-peaked Gaussians into wider-peaked Gaussians), and so on.

Aspects of mathematical sense-making have already been studied in introductory physics [20-24], and to a lesser extent in upper division classical physics. We ask: What does mathematical sense-making look like in quantum mechanics?

This paper is not an empirical investigation meant to map the terrain of students' mathematical sense-making in quantum mechanics, nor an experiment to test particular hypotheses. Instead, it is a theory-building effort which uses data to illustrate the constructs and conjectures we introduce and to begin exploring how researchers can "see" 
mathematical sense-making in students' QM problem solving. We hope this work will be generative for two overlapping pockets of physics education research (PER). One is research about student thinking and associated curriculum development in upper division quantum mechanics. The other is research on students' mathematical sense-making in physics, which has previously focused mostly on students in introductory physics courses, with some exceptions $[13,25]$. A smaller literature touching on mathematical sense-making in upper division physics has, for instance, catalogued misconceptions and knowledge gaps students display when interpreting fundamental equations in QM [26]; identified mistakes students make when translating between charge distributions and delta functions or vice versa, often arising from misunderstandings of notation and from inappropriately pattern matching to simple one-dimensional cases [27]; found that students have trouble making physical sense of the derivation for and meaning of the Boltzmann factor [28]; and identified constructs within students' personal epistemologies that are associated with mathematical sense-making or its absence $[22,25]$. However, most of this previous work on upperdivision physics reasoning does not identify particular cognitive resources that could serve as a bridge between students' prior knowledge and a fully correct conceptual and symbolic understanding of the targeted material.

In this paper, we make plausible the argument that the cognitive resources needed for mathematical sense-making in introductory physics are necessary but not sufficient for mathematical sense-making in quantum mechanics. To start, we briefly review a theoretical construct for describing and analyzing aspects of students' mathematical sensemaking in introductory physics, Sherin's symbolic forms [29], and review how it captures facets of emerging expertise in problem-solving. Next, we present an episode of two students collaboratively answering a quantum mechanics question. We argue that the students engage in some aspects of mathematical sense-making but not others, and that this sense-making (and lack thereof) is consequential in their reasoning, not just a tangent and not just "along for the ride." Then, using this episode as a touchstone, we introduce some new candidate symbolic forms relevant to eigenvector-eigenvalue equations, arguing for their existence and importance in QM mathematical sense-making. We also discuss how the relation between symbolic forms and physics concepts is likely to be more indirect in $\mathrm{QM}$ than in introductory physics.

Finally, stepping back from these finer-grained details, we argue that when students do not succeed in mathematical sense-making in quantum mechanics, the root cause is not necessarily a more general failure to engage in mathematical sense-making, or an epistemological belief that mathematical sense-making is not productive in this domain [30]. The issue may instead be that students are not using particular cognitive machinery, which we model as particular symbolic forms, needed to engage in expert mathematical sense-making in $Q M$ (and perhaps in other fields of physics using the same mathematical constructs). Some of these new symbolic forms, we argue, are not primitive, but are built out of smaller components, including some existing symbolic forms. There could, of course, be social, epistemological, or conceptual reasons for why this machinery is not engaged in specific scenes of collaborative physics work, but we do not explore these reasons here.

\section{MATHEMATICAL SENSE-MAKING IN PHYSICS}

Mathematical sense-making is not a single, well-defined construct. Different flavors of it have been discussed, using varied terminology, in the mathematics and science education literature. A common thread, however, is to distinguish mathematical sense-making from an algorithmic approach to conceptualizing and approaching problem solving. An early example of this distinction comes from Wertheimer [31], who posed problems of the form $(812+812+812+812+812) / 5=$ ? Many students successfully took an algorithmic approach, summing up the numerator and dividing by the denominator to get 812. But other students saw the "shortcut," based on understanding the conceptual meaning of repeated addition and of division.

\section{A. How mathematical sense-making differs from standard problem solving}

Researchers studying mathematical sense-making in physics typically focus on reasoning in which students not only leverage a conceptual understanding of the relevant mathematical entities and operations, as in the Wertheimer example above, but also connect the meaning of those entities or operations to causal and/or functional relations in the physical world. In doing so, they (like Wertheimer) contrast mathematical sense-making with approaches in which conceptual reasoning and symbolic manipulations are more cleanly separated.

To illustrate these points, we summarize an example from Kuo et al. [23]. They studied students' responses to the following question:

Suppose you are standing with two tennis balls on [a high balcony]. You throw one ball down with an initial speed of 2 meters per second; at the same moment, you just let go of the other ball, i.e., just let it fall. What is the difference in the speeds of the two balls after 5 seconds - is it less than, more than, or equal to 2 meters per second? [If the student brings it up, the interviewer says to neglect air resistance]

Kuo et al. found that students' responses often fit into one of two patterns. In the first pattern, many students' 
solutions could be summarized, at least approximately, by the following steps.

1. Draw and label diagrams of the two separate balls.

2. Decide what physical principles are relevant or what "kind" of problem it is, in this case a kinematic constant-acceleration scenario with gravity accelerating both balls at the same rate $(g)$,

3. Translate the physical description and insights from steps 1 and 2 into equations-in this case, $v=v_{0}+a t$ applied to each ball separately

4. Solve the equations from step 3 , in this case to solve for the final speeds and then the difference between them.

5. Check the final answer for plausibility (a reflection step, in this case often prompted by the interviewer).

A principled, metacognitively aware version of these steps, with an emphasis on carefully thinking through the physical scenario and the underlying concepts or principles before jumping to equations, corresponds to expert quantitative problem solving as described, taught, and researched by many in PER [20,21]. Potential opportunities for mathematical sense-making arise when students "translate" the physical scenario into equations (step 3) and check the final answer for plausibility (step 5). In this particular case, however, most students' step 3 was simply a matter of recognizing this scenario as a free-fall kinematics problem, for which they can use the "standard" constant-acceleration kinematic equations with $a=g$; students following this solution path did not try to figure out the deeper "meaning" of the equation(s), which simply served as problem-solving tools. Step 5, by contrast, sometimes did provoke mathematical sense-making, as we now describe.

Although most students in Kuo et al.'s study took the solution path outlined above, some students approached the problem differently, either right from the start or when revisiting their answers in step 5. In this second approach to the problem, students used some variant of the following reasoning, explicitly referring to the equation $v=v_{0}+a t$ : While falling, both balls speed up at the same rate. Therefore, they both gain the same amount of speed. So, since the thrown ball starts out $2 \mathrm{~m} / \mathrm{s}$ faster than the dropped ball, the thrown ball is still $2 \mathrm{~m} / \mathrm{s}$ faster at the later time. Kuo et al. argued that this solution path relies on mathematical sense-making, in that students are using the conceptual meaning of the equation: the final velocity is the initial velocity plus the velocity gained (as given by the rate of gain of velocity multiplied by the time over which the gain occurs). Following Wertheimer, they also argued that this "shortcut" approach reflects a form of adaptive expertise [32] with respect to using equations, a form of expertise that physicists develop and value.

\section{B. Symbolic forms}

In this section we review the cognitive structures that Kuo et al. and others [33] have used to model the knowledge upon which students draw when engaged in mathematical sense-making.

Sherin [29] videotaped pairs of 3rd semester physics students working together to address unfamiliar physics scenarios, ones for which they could not simply use a standard problem-solving algorithm. Using a "bottom up" approach to characterizing students' knowledge and reasoning, Sherin proposed the existence of knowledge elements called symbolic forms. Each symbolic form has two components: a symbol template and an associated conceptual schema. To illustrate this idea, we discuss the symbol template that Kuo et al. used to cognitively model students' mathematical sense-making in the problem discussed above, namely, base + change [23,29].

The symbol template for base + change is $\square=\square+\square$ : a quantity equals the sum of two other quantities. And more generally, a symbol template is an algebraic expression or equation with "blanks" instead of particular values filled in. A conceptual schema, by contrast, is an intuitive schema for thinking about the physical world. The conceptual schema for base + change is the intuitive idea that the amount you end up with is the amount you started with plus the amounts gained (or lost). This final amount is initial amount plus change conceptual schema appears in people's reasoning in everyday life, reasoning about money or pizza, sometimes without quantitative precision (e.g., "I just got paid so I have more money than before!"). In Sherin's schema, the base + change symbolic form is a cognitive "fusion" of the $\square=\square+\square$ symbol template and the final amount is initial amount plus change conceptual schema into a "compiled" cognitive unit that the student then uses in a unified way, without teasing apart the symbolic template from the conceptual schema. For instance, in the mathematical sense-making approach to the problem discussed above, students parse " $v=v_{0}+a t$ " as the symbol template $\square=\square+\square$ and reason in terms of the final amount is initial amount plus change to interpret the equation as saying "final velocity equals the initial velocity plus the change in velocity." By this account, the base + change symbolic form has "dual citizenship" in the world of formal symbolic reasoning and the world of intuitive reasoning about physical reality.

Importantly, in Sherin's framework, a given symbolic template can be paired with multiple conceptual schema, producing multiple symbolic forms. For instance, the symbol template of base + change, $\square=\square+\square$, can also be paired with a whole consists of parts conceptual schema - the idea that a thing can be broken into parts, and conversely, that parts can be combined into a thing. People might apply that conceptual schema even when reasoning nonquantitatively, e.g., "I'm extra stressed today because I lost my phone and I have a deadline at work." But when the whole consists of parts conceptual schema becomes cognitively coupled to the $\square=\square+\square$ symbol template, the result is the parts of a whole symbolic form. As Kuo et al. 
discuss, students might use this symbolic form when writing an equation for the total energy of a system as the sum of kinetic and potential (and possibly other forms of) energy. (Note that the more general symbol template for parts of a whole is " $\square=\square+\square+\square+\cdots$," reflecting the idea that a whole can consist of more than two parts.)

Other research has also documented mathematical sense-making that could be modeled in terms of symbolic forms, though without the explicit modeling. For instance, Bing and Redish [25] analyze a student (in an interview) figuring out how to modify a fluid-flow "mass conservation" equation to apply to a situation where density refers to the concentration of a chemical created at rate $Q(\mathbf{r}, t)$. The student writes down an equation that we can schematize, based on the student's previous comments, as

$$
\begin{gathered}
\pm Q-\text { rate of change of the amount of chemical inside the volume }] \\
\quad=\text { rate of flow of chemical out of the boundary of the volume }]
\end{gathered}
$$

Note that the two terms represented by brackets involve derivatives and volume integrals, but their exact form does not matter for the point we are making here. Nor does it matter, for our argument, that the student neglected to integrate $Q$ over the volume.

The student wrote " \pm " because he was not sure which sign to use. At the interviewer's suggestion, he thought through whether $+Q$ makes sense in the equation. He initially (and correctly) thought it might:

Uhhh, yeah, if it's a, if it's a positive sign then the right hand side has to increase (points to right-hand side of equation) because something is getting sourced inside this volume. So for this to increase... (points to his drawing, representing the physical meaning he attributed to the term on the right-hand side of the equation)

The student is thinking that, the faster the chemical gets created ("sourced") inside the volume (bigger $Q$ ), the faster it must flow out of the volume (bigger right-hand side of equation). Then the student looks back at the equation and decides $Q$ must have a minus sign in front:

Yeah, so it cannot be a positive, it has to be a negative, because then that's [the right-hand side] going to increase-for these signs to match (points to the signs in front of the two terms on the left-hand side) for the magnitude [of right-hand side] to increase. (Erases “ \pm ” and writes a minus sign in front of the $Q$ ). So it's probably negative.
Although the student's meaning is ambiguous, we see the following interpretation as plausible: In this moment, the student sees the two terms on the left side as "contributors" to or influences upon the overall rate of flow out of the volume, as represented by the right side. The student decides that the signs of those two contributions or influences must "match"; and negative is ok because now the student is thinking about what it takes for the "magnitude to increase" on the right side (emphasis ours).

So, we suggest that the student rejects the equation in the form $+Q-[]=[]$ because in this moment he interprets the left side using the opposition symbolic form, the idea that two influences oppose each other rather than helping each other, corresponding to the symbol template " $\square-\square$ "one term subtracted from the other. By matching the signs on the left-hand side, he "fixed" the opposition, expressing instead that the two contributions or influences help or reinforce each other. (Bing and Redish do not interpret the data presented in terms of the machinery of symbolic forms; here, we are reinterpreting the data through the lens of symbolic forms. However, their analysis is wellaligned with and does not contradict the symbolic forms interpretation.)

From an expert perspective, the student's reasoning went wrong because he interpreted the second term on the left side, [rate at which amount of chemical inside the volume changes], as an influence on the flow rate out of the volume rather than the net effect of other influences. Had the student rearranged the equation as

$$
\begin{gathered}
\pm Q-[\text { rate of flow of chemical out of the boundary of the volume }] \\
\quad=[\text { rate of change of the amount of chemical inside the volume }]
\end{gathered}
$$

and activated opposition to interpret the left side, he might have correctly concluded that $Q$ should be positive since the "sourcing" of chemical and the outflow of chemical are opposing influences that determine the net rate at which the amount of chemical in the volume increases.

We present this complicated, ambiguous case to illustrate that symbolic forms, though "discovered" in introductory physics, can help us describe and analyze students' mathematical sense-making in classical upper-division physics, too.

Based on his particular data corpus, Sherin generated a "semiexhaustive list" of 21 symbolic forms but acknowledged that others undoubtedly exist. Later in this paper, we will propose new symbolic forms to model aspects of 
students' reasoning about quantum mechanics. Our main point for now is that symbolic forms are a way of cognitively modeling an aspect of mathematical sense-making, the "blending" (fusion) of formal or symbolic and intuitive or conceptual reasoning. This kind of blending contrasts sharply with a plug-and-chug approach to problem solving. It also contrasts somewhat with exemplary quantitative problem solving, as articulated by Heller and colleagues [20], where mathematical sense-making sometimes occurs when the physical scenario is "translated" into equations, depending on how that translation is conducted, and sometimes when the answer is checked for sensibility, again depending on the nature of that check. The slice of mathematical sense-making we explore in this paper takes place during the "mathematical" steps, not just before and after.

\section{Summary and next steps}

So far in this paper, we have reviewed how symbolic forms can be used to describe and analyze an aspect of students' mathematical sense-making, the blending of formal or symbolic and intuitive or conceptual reasoning. We reviewed arguments that this aspect of mathematical sense-making is not automatically part of "good" quantitative problem solving as portrayed in the PER literature, but that mathematical sense-making is part of physicists' adaptive expertise in the use of mathematics.

Now we turn to the central issue of this paper, the ways in which mathematical sense-making might look the same and/or different as described above when students grapple with quantum mechanics. To ground our later theorybuilding discussion in a specific example, we first present a pair of students addressing a quantum mechanics question.

\section{METHODOLOGY}

\section{A. Data collection}

We videorecorded two students in a clinical (nonclassroom) setting in March 2015, working collaboratively through the beta version of a new tutorial on the "particle in a box" (PIAB) [10,34,35]. Fred and Gus (pseudonyms) were undergraduate electrical engineering (EE) majors recruited via email from Physics of Devices, an upperlevel EE course with significant quantum mechanics content. Fred and Gus were in different sections of Physics of Devices but knew each other from other courses. The students were given the tutorial, asked to work on it together, and encouraged to talk out loud about their thinking.

\section{B. Data analysis}

The episode presented here immediately stood out to the interviewer (the first author) as notable, so he brought it to the full group of authors for collaborative video viewing and analysis [36]. This episode was "found data" in the sense that investigating mathematical sense-making was not an original objective in collecting the data; we were focused on the dynamics of students' ontological conceptions. However, the relevance of the data to exploring mathematical sense-making emerged through the collaborative analysis.

Even though we have facilitated three other focus groups using the same tutorial and collected many more hours of video of students working through related tutorials, there are no other episodes in our data corpus in which mathematical sense-making is so visible. This is likely because the tutorials emphasize qualitative reasoning and do not prompt the use of equations; Fred and Gus bring in an equation on their own. Other students doing the same tutorial responded to the same prompts with purely conceptual reasoning, and, therefore, mathematical sensemaking was not a step that they would logically have taken even if those students would have been inclined to do so under other circumstances.

So, we are not claiming that Fred and Gus are a representative sample. Indeed, we are claiming the opposite; we picked them because they were atypical, "spontaneously" engaging in equation-based reasoning while working through the conceptual tutorial. Therefore, the value of this study is in its potential theoretical generalizability [37]: this single instance can inform the way we think about mathematical sense-making more generally, by presenting a counterexample that challenges existing frameworks. In other words, this study facilitates theory building in which we posit cognitive constructs and relations among them whose existence and prevalence can be tested in future studies. While gathering more data on mathematical sensemaking in quantum mechanics would be a valuable future research direction-indeed, that is one of our points-the conclusions in this paper, which are theoretical conjectures, do not depend on having a larger data set.

The selected episode lasts 13 minutes and consists of Fred's and Gus's discussion in response to a single prompt in the tutorial (including the interviewer's follow-up questions). This episode ends when the students move on to the next prompt. However, we viewed the full session to see whether the material before and after the episode of interest could further inform the analysis.

Our analysis is grounded in a "resources" theoretical framework [38-40] that assumes students' thinking can be dynamic and fragmented, with different "conceptions" and different epistemological stances "turning on" in response to different contextual cues. We therefore attend to the dynamics of student thinking on a short time scale, down to individual conversational turns and even within turns, rather than averaging over the whole episode to attribute conceptions and beliefs to particular students.

\section{CASE STUDY}

Before the selected episode began, earlier prompts in the particle-in-a-box (PIAB) tutorial had asked the students to 
consider a one-dimensional PIAB (infinite square well) in the ground state. The students were asked to sketch a graph of the ground-state wave function, which they did correctly. Question 4 then asked, "If you were to measure the position of the particle at some point in time, what position(s) would you expect to measure? Why? Will you get the same measurement every time?" Fred and Gus responded that the wave function represents the probability of finding the particle at each point, and so different measurements will yield different positions, in accordance with this probability distribution.

\section{A. Does energy depend on position?}

The episode that we analyze is in response to the next prompt, question 5:

If you were to measure the energy of the particle at some point in time, what would you expect to measure? Why? Will you get the same measurement every time?

Fred's initial response is “So, isn't the energy constant?" We do not have enough information to determine where this is coming from. Fred could be applying (correctly in this case) a principle from classical physics (conservation of energy), or he could be basing this conclusion on quantum mechanics (the system is in the ground state, which has a defined energy). But in either case Fred seems to be applying a general principle, as intended by the tutorial prompt, rather than performing a calculation.

Gus does not take up Fred's proposal. Instead, he asks "What's the full psi equation?" and attempts to reconstruct the time-independent Schrödinger equation, with Fred's cooperation:

Gus: (mumbling) Minus hbar squared over $2 \mathrm{~m}$ (pause) partial, um, is it $\psi$ of $x$, or partial $\psi$ of partial $x$ ?

Fred: Is this the one [inaud.] the partial derivative of $E$, like, respect to $k$, or something like that? (Fred makes a face; Gus doesn't respond, but continues looking at his paper) Maybe that was just some other thing I was doing. (puts his face in his hands)

Gus: ...plus $V$ of $x \psi$ of $x$ equals $-I$ think it equals an energy?

Fred: That sounds about right.

Gus: But I know there's something in here (writing).

Fred: Ok, I see what you're talking about. Is that the derivative of $\psi$, maybe?

Gus: Oh, V of $x$ times the derivative of $\psi$ ?

Fred: No, I'm saying, that (pointing), where you're, the part we're missing.

Gus: Yeah, is it derivative or partial derivative? $d \psi d x$, or partial $\psi$ partial $x$ ?

Fred: I think it's just derivative.

Gus: (mumbling) $d \psi d x$.
The equation that Gus writes down in the end is

$$
-\frac{\hbar^{2}}{2 m} \frac{d^{2} \psi}{d x^{2}}+V(x) \psi(x)=E
$$

This is almost correct, but has $E$ on the right side instead of $E \psi(x)$. (This same error has been documented by Singh [26].) This change, likely based on an error of recall, influences the meaning of the equation: instead of an eigenvalue equation for $\psi(x)$, which can be used to find eigenfunctions and eigenenergies, it appears to be simply an algebraic expression to solve for $E$. From this version of the equation, it appears that the energy depends on the wave function $\psi(x)$, and Fred and Gus already found in previous questions that the wave function varies with $x$. Fred keys into this and says "Actually, maybe it [energy] wouldn't be constant then." Thus, he is revisiting his previous conclusion about the energy based on what the equation appears to show.

Gus wonders whether this means that the wave function is time dependent, and asks the interviewer for clarification:

Gus: Well, so, when we look at number 5, should it just be like $\psi$ of $x$ (turning to interviewer), or should it be $\psi$ of $x$ and $t$ ? Or would that be giving away too much? Interviewer: Well, we're-it's asking about the energy. So if you were to measure the energy, what would you expect to measure, so same question as 4 , but 4 was asking about measuring the position, and now we're asking about measuring the energy.

Gus: Oh, ok. Kind of the same as the previous one, which is-

Fred: Yeah, because the energy depends on the wave function. And since the wave function is never, like it's not, never like the same at any point, like it has different values, so the energy would depend on the position? Of the particle? Would it? Does that make sense?

Gus: Well, we are taking, like, $\psi$ and $V$ at a certain, like, $x$ coordinate, and [inaud.] understand why this is 0 to $L$, so like $V$ of $x$ here, or $V$ of $x$ here, would be different from V of x / Fred: Yeah / Gus: here.

We can model part of Fred and Gus's reasoning here in terms of the dependence symbolic form, whose symbol template is simply that a quantity is a function of another quantity and whose conceptual schema is the idea that one entity or process is causally or structurally linked to another (e.g., "the more I get behind schedule, the more anxious I become."). Gus and Fred are looking at the equation and concluding that, since the energy is a function of $\psi$, and $\psi$ is a function of position, the energy is therefore a function of position. Therefore, the energy of the particle does not have a fixed value, since the particle's position does not have a fixed value. Based on this mathematical sensemaking, the students reject Fred's original idea ("isn't the energy constant?") in favor of drawing a conclusion from 
the equation. The equation they wrote down is incorrect, so it is no surprise that their mathematical sense-making leads to an incorrect conclusion. Our point here, however, is that they are mathematically sense-making instead of just "plugging and chugging" or performing symbolic manipulations. In other words, rather than using the equation as merely a computational tool, they examine the functional dependencies in the equation to draw conclusions (albeit uncertain ones) about which physical parameters depend on which other physical parameters, linking those mathematical dependencies to physical dependencies for the PIAB.

\section{B. Questioning their conclusion}

Fred and Gus do not simply accept the answer they reached so far, based on "mathematical" reasoning, as correct. They question whether they have reached the right conclusion:

Fred: It's just a weird concept to think that the energy is / Gus: Yeah / Fred: dependent on the position. (pause) Is it? (pause) I mean, according to that equation it is.

Gus: Yeah, if that's the right equation, then yeah. Oh yeah, I think it's 'cause usually when we do these infinite well problems, we assume like $V$ equals 0 here, $V$ equals $O$ here, and then $V$ equals like 5 electron volts or something inside. So that's probably why you just assume.

Fred: Ok.

Gus: 'Cause V, or-yeah, V will be the same here, but I think $\psi$, since $\psi$ is different along this curve, even though $V$ is the same, the energy might change. [Inaud.] wonder if I'm just like pulling this out of thin air. But, um-

Fred: I'm good with saying that, that it would be the same because it depends on the wave function.

Fred is torn between (i) the result that, "according to that equation," the energy depends on position, and (ii) his earlier result based on either physical intuition and/or the conceptual principle that the energy is constant. While he does not dispute that the equation leads to this result, he is uncomfortable with it, saying that it is "just a weird concept." Gus has lingering doubts about whether "that's the right equation" or whether he is "just like pulling this out of thin air."

In this segment, we see Fred and Gus continuing to invoke dependence ("since $\psi$ is different along this curve... the energy might change") but also engaging in facets of mathematical sense-making different from what we discussed above. First, instead of simply accepting their mathematically based conclusion as correct, they acknowledge that they might have made a mathematical error ("wonder if I'm pulling this out of thin air"). Although seemingly mundane, this kind of questioning of mathematical conclusions does not always occur when students solve physics problems [22,41]. And relatedly, as the discussion continues, the students explicitly connect epistemological issues concerning the (counter)intuitiveness of quantum mechanics to whether they should trust counterintuitive mathematically based conclusions:

Interviewer: What would you expect the-if, if it weren't for that (the mathematically based conclusion that energy depends on position), like what would be more intuitive?

Gus: Um-

Fred: I would think like the energy would be constant at each $n$ level. Like $n$ equals 1 has like a constant energy value, um, pertaining to it, $n$ equals 2 has like a constant energy value pertaining to it. That's what it seems like it would be to me.

Gus: Kind of like if you're like at a certain height and you drop the object (gestures the sides of a building), it would have a certain amount of like kinetic energy as it's falling-or, a certain amount of potential energy (correcting himself), then if you went up another floor of the building (gesturing) it would have a certain amount of potential energy (gesturing) /Fred: Right./ Gus: Like even if you went from this side of the building (gesturing) to the back side of the building (gesturing), it would still have the same amount of potential energy /Fred: Right./ Gus: if you're holding it above the ground.

Fred: Mm hmm. But hey, quantum mechanics doesn't make sense a lot of the time. (laughs)

Greg: (mumbles)

Fred: So we want to just go with that? /Gus: Yeah./ Like the energy depends on the position? Like the wave function?

Gus: Yyyes. So if we write down the equation, maybe we'll get partial credit. (both laugh)

Gus invokes an energy-as-vertical-location metaphor $[42,43]$ to develop a classical analogy supporting Fred's idea that a particular constant energy should "pertain" to each $n$ level. Fred endorses the analogy ("Mm hmm") but then makes an epistemological claim: "quantum mechanics doesn't make sense a lot of the time." Fred and Gus then use that epistemological warrant to "just go with that," the equation-based conclusion that "the energy depends on the position." Their laughter and jokes about earning "partial credit" suggest that they do not see their conclusion as completely solid.

So, in this segment, we see Fred invoking an epistemological view about quantum mechanics-“[it] doesn't make sense a lot of the time"-as a warrant for dismissing their physical intuition when it conflicts with their counterintuitive equation-based conclusion. While this attitude is sometimes expressed by introductory students about physics in general, it is also exhibited in ways specific to quantum mechanics even among more advanced students and experts $[4,44,45]$. Reasonable physicists can disagree 
about the extent to which quantum mechanics doesn't make sense and therefore how carefully one should question and recheck a given unexpected conclusion derived from mathematics. In any case, now that the students have reached an impasse, the subsequent dialogue suggests that part of Fred's motivation may be to take an escape hatch [34] to close the conversation. Our point here is that Fred and Gus are considering, at all, for whatever reasons, the broader issue of to what extent mathematically derived results should (or should not) make sense in quantum mechanics. Whether we categorize this reasoning as an aspect of mathematical sense-making or as something linked to mathematical sense-making - perhaps "epistemic cognition about mathematics" - our point is that it instantiates a form of sense-making that, when further developed, can contribute to expert learning and problem solving in quantum mechanics. That is why we have flagged it in this paper.

\section{Dependence redux}

At this point, the interviewer decides to correct the equation to see whether this will assist Fred and Gus in reconciling the equation with their intuition:

Interviewer: All right, so I think, I was going to tell you that in the equation, so the issue there, on the right side of the equation, it's gonna be E times $\psi$ on the other side. /Fred: Mmmm!/ 'Cause that's the eigenvalue, so you do stuff to $\psi$, it equals to this value times $\psi$.

Fred: So even if we divide this over, /Gus: Well.../ it would still be-it would still be dependent on the $\psi$.

Gus: I mean, well, we could divide, but that would just be 1 over $\psi x$ here.

Fred: Would it? What is the derivative of something divided by its actual (pause) the derivative of $\psi$ divided by $\psi$ ?

$[\ldots]$

Fred: But I feel like it would still depend on the wave function, right?

Gus: Seems like it. 'Cause this is $E \psi$ of $x$ on the right side. Hmm. (pause) Yeah. [...] So this is the derivative, first part is the derivative of $\psi$ of $x$, second part the $V$ of $x$ is still times $\psi$ of $x$, which is still dependent on $\psi$ of $x$, and this energy is still dependent on $\psi$ of $x$.

Fred: You [inaud.] isolate the energy. There's still gonna be a $\psi$ of $x$ in the equation somewhere.

The interviewer's intervention has little impact on the students' reasoning and conclusion. The inclusion of $\psi$ on the right side of the equation simply leads them to divide both sides by $\psi$ to "isolate the energy," and they still find that the energy depends on $\psi$, which depends on $x$. So, the students continue to interpret this equation with the Dependence symbolic form, treating the equation as a regular algebraic equation, not an eigenvalue equation.

\section{DISCUSSION: MATHEMATICAL SENSE-MAKING IN QM}

In this section, we use Fred's and Gus' reasoning as a launching pad to begin building theory about what mathematical sense-making might look like in quantum mechanics. As emphasized above, we offer constructs and conjectures not as "proven" but as ideas to be refined and tested in future work. Fred and Gus inspire and illustrate but do not provide empirical support for these ideas.

\section{A. The intersection of epistemology and mathematical sense-making}

Previous work discussing how physics and engineering students' epistemological stances affect (and are affected by) their use of mathematics has mostly (but with some exceptions) been contextualized to lower-division courses. These case studies have mostly highlighted cases where epistemological stances contributed to students' "overtrusting" their (mistaken) mathematical reasoning at the expense of everyday intuitions, or conversely, trusting their intuitions and revisiting the (incorrect) mathematics to attempt a reconciliation $[30,41,46-49]$. So, in this body of work, trusting math to the detriment of physical reasoning is generally the "unsophisticated" epistemological stance.

In quantum mechanics, by contrast, many of our everyday physical intuitions - that particles have well-defined trajectories, that the lowest possible kinetic energy a particle can have in a given scenario is zero-are violated. So, students (and experts) can reasonably argue that, in certain quantum mechanical contexts, it is epistemologically sophisticated to "just trust the math" to the detriment of physical reasoning more often than might be the case in, say, classical kinematics. And at a more meta level, the value of students stepping back and thinking about this epistemological issue may be higher in quantum mechanics than in more "intuitive" branches of physics. In other words, we see value in the "mathematical sense-making" (or whatever it should be called) illustrated in Sec. IV. B. In QM learning and problem solving, carefully playing out a mathematical argument and also a conceptual argument, and then using epistemological considerations to help choose between those two paths when they cannot be reconciled, can be more productive than just choosing one path or the other. Or so we hypothesize.

\section{B. Productive "classical" symbolic forms}

We hypothesize that many of the symbolic forms identified by Sherin, though generated in an introductory physics context, can also support mathematical sensemaking in quantum mechanics. Fred and Gus' dependence-based reasoning was unproductive when applied to an eigenfunction-eigenvalue equation, but would likely be productive in other QM scenarios. For instance, consider 
the potential energy expression for an electron orbiting a nucleus, $V(r)=-Z e^{2} / r^{2}$. Dependence-based reasoning applied to this expression could help students understand why higher-energy orbitals correspond to higher $r$, i.e., to wave functions for which $\langle r\rangle$ is greater. Similarly, the base + change symbolic form can be productive in a number of contexts in quantum mechanics. One example is perturbation theory, where the Hamiltonian might be written in a form such as $\hat{H}=\hat{H}_{0}+\hat{H}_{1}$, where $\hat{H}_{0}$ (the "base") represents the Hamiltonian for an "unperturbed" system for which the Schrödinger equation has a known solution, and $\hat{H}_{1}$ (the "change") represents a small perturbation to that Hamiltonian. Even if this equation is written with different symbols (e.g., $\hat{H}=\hat{H}_{0}+\lambda \hat{V}$ ), the base + change structure is still evident, and it can be productive to conceptualize the eigenfunctions of $\hat{H}=$ $\hat{H}_{0}+\hat{H}_{1}$ as the "base" eigenfunctions (of $\hat{H}_{0}$ ) modified by a small "change."

So, our conjecture here has two parts. First, we conjecture that symbolic forms can be used to describe and analyze some aspects of mathematical sense-making in quantum mechanics. And second, we suspect that many of the previously identified symbolic forms play a role in that sense-making; as researchers we do not need to identify an entirely new collection of forms.

However, some new symbolic forms are needed, as we now discuss.

\section{New symbolic forms for QM mathematical sense-making}

Fred and Gus' reasoning in Sec. IV.C inspires us to hypothesize that productive mathematical sense-making in QM includes previously unidentified symbolic forms that contribute to understanding eigenvalue-eigenvector equations.

We are not the first to focus on eigenvalue-eigenvector equations as a source of student difficulties. Thomas and Stewart [50] argued that such equations are particularly difficult to understand conceptually because the two sides of the equation, $A x=\lambda x$, represent two different processes on the same object. They showed that students continue to think about these equations symbolically and procedurally, rather than conceptually. In other words, students do not generally mathematically sense-make when using eigenvalue-eigenvector equations. Fred and Gus, by contrast, are engaging in mathematical sense-making, but using a symbolic form (dependence) that may both reflect and reinforce their treatment of the equation as a "regular" algebraic equation. This locally coherent pattern of reasoning may help to explain why the interviewer's intervention, in which he not only corrects the Schrödinger equation but also reminds the students to think of $E$ as an eigenvalue, has little effect on the students. It might also be the case that the students do not yet have in their cognitive ecologies certain tools that could help them make better sense of the time-independent Schrödinger equation, which is an eigenvalue-eigenvector equation. In the remainder of this section, we explore what those tools might be, representing them as symbolic forms.

\section{The transformation symbolic form}

To make sense of eigenvalue-eigenvector equations and other QM formalism, students must understand that an expression such as $\hat{H} \psi$ or $\hat{S} \phi$ represents not multiplication, but rather, an operation on a vector or function that transforms it into another vector or function. We propose representing this understanding of "operating on a state" as follows. The symbol template is $\hat{\square}|\rangle$, where $\hat{\square}$ is an operator and |\rangle is a state, represented as a vector, function (e.g., in position or momentum space), or abstract ket. The corresponding conceptual schema is reshaping, the intuitive idea of "stuff" getting molded into a different shape. So, an operator acting on a vector "spits out" another vector that in general points in a different direction. Or, an operator acting on a wave function spits out a new function with a different shape. This reshaping conceptual schema, when coupled to the $\hat{\square}|\rangle$ symbol template, constitutes the transformation symbolic form. We conjecture that transformation is part of expert physicists' cognitive ecologies and that students can learn it, though doing so is not easy.

Whereas Sherin's original symbolic forms activated in classical physics scenarios often lead to a ready physical interpretation (e.g., final velocity is initial velocity plus change in velocity), transformation in the quantum context is less directly connected to physical entities. For instance, the momentum operator acting on a state, $\hat{p}|\rangle$, may not have an obvious physical meaning on the surface, though if the vector resulting from this operation is expressed in the momentum basis, it can be understood as a list of possible measurement outcomes with associated probabilities. Taking the inner product of this entity with the corresponding "bra," \langle| , we obtain a different physical thing, the expectation value of momentum. In the common, special case of the Hamiltonian acting on a wave function, the transformation $\hat{H} \psi$ is proportional to the rate of change of $\psi$, a mathematical function that must further be manipulated to yield the wave function at a later time. (In other contexts, the transformation symbolic form has different meanings. For example, if a rotation matrix acts on a vector, the result is the same kind of vector but pointing in a different direction.) Further research is necessary to understand how students see the relationship between these different instantiations of transformation.

So, transformation illustrates a conjecture we now put forward: "new" symbolic forms needed for mathematical sense-making in QM, as compared to the "old" symbolic forms Sherin catalogued in introductory physics, will typically be a step farther removed from physical interpretations. 


\section{Eigenvector-eigenvalue symbolic form}

Here we propose the existence of a new symbolic form, eigenvector eigenvalue. While this type of equation is of course nothing new to physics faculty or advanced students, it represents a new addition to the symbolic forms framework in both content and structure. Its symbol template is

$$
\hat{\square}|\rangle=C|\rangle \text {, }
$$

where the state vector or function is the same on both sides and where $C$ is a constant with (or without) units. The conceptual schema is complex because both sides of the equations are, separately, symbolic forms. The left side is the transformation form we just discussed. The right side is the coefficient form from Sherin's original study, in which a quantity interpreted as a constant is multiplied by a quantity interpreted as changeable, and with the constant interpreted as a coefficient-something that "tunes" or moderates the changeable quantity. ${ }^{1}$ This difference between the symbolic forms corresponding to the right vs left side mirrors Thomas and Stewart's [50] insight that eigenvector-eigenvalue equations are difficult for students because the two sides correspond to two different processes on the same object. The equals sign in the equation also has a specific meaning: for a given operator, there are particular values of $C$ and |\rangle that satisfy this condition of equality and are solutions to the eigenvalue equation. This is different from other meanings that the equals sign can have [51,52], such as definition or "happens to be the same."

The conceptual schema corresponding to the eigenvector-eigenvalue form as a whole is transformation that reproduces the original, the idea that a potentially transformative process has a net effect of reproducing the original state of affairs. This conceptual schema underlies the sense that the following two scenarios are similar in a way: Trying to hike out of a woods but ending up back where you started (perhaps with more mud on your boots), and trying to scour scratches off a bowl but creating as many new scratches as are removed.

The eigenvector-eigenvalue symbolic form constituted by the coupling of the above symbol template to transformation that reproduces the original supports the following interpretation of an eigenvector-eigenvector equation: Each solution to the equation is a state vector or function paired with a coefficient such that the operator acting on

\footnotetext{
${ }^{1}$ Another symbolic form called scaling has a similar symbol except the constant is unitless and is interpreted as a "resizing" factor. In most eigenvalue-eigenvector equations commonly used in $\mathrm{QM}$, the " $C$ " corresponds to a physical quantity with units, such as energy or momentum or angular momentum, and hence cannot be correctly interpreted as simply resizing the state vector or function. However, it's possible that a student thinking of the right side in terms of scaling rather than coefficient could still engage in productive mathematical sense-making about the eigenvector-eigenvalue equation as a whole.
}

that state spits out the same state multiplied by the coefficient. And therefore, states for which the operatorinduced transformation does not "reproduce" the original state are not solutions.

Expert reasoning with eigenvector-eigenvalue equations in quantum mechanics adds layers of physical interpretation on top of this conceptual understanding of the equation: (1) The operator corresponds to a physical quantity such as energy or momentum, (2) the solution states correspond to eigenstates of that quantity-states for which the physical quantity has a definite value, a value given by the eigenvalue (coefficient).

In Fred and Gus's interview, once the interviewer corrected the time-independent Schrödinger equation, activation of eigenvector eigenvalue in the students' reasoning might have disrupted their treating $E$ as something that could be variable or constant, depending on the functional relations on other side of the equation. That symbolic form might have supported their conceptual conclusion that $E$ - the eigenvalue in that equation-must be constant for a given "allowed" state such as the ground state. In other words, what is leading the students astray is not the absence of symbolic forms in their reasoning about the time-independent Schrödinger equation, but which symbolic form they employ. They are not identifying it as an eigenvalue equation and therefore not attending to the varied ontologies of the mathematical objects in the equation (scalar constants, states, and operators) and the relations among them specified by eigenvector eigenvalue.

\section{Differences between the "old" and "new" symbolic forms}

In this section we briefly highlight two differences between the old symbolic forms from Sherin's original study and the new forms proposed above.

\section{Directness vs indirectness of physical interpretation}

The old forms such as base + change and parts of a whole, when activated within a particular classical context, invite a ready, even obvious physical interpretation. For instance, parts of a whole applied to an expression for the total energy of a system immediately invites a physical interpretation of the different terms as "contributions" to the overall energy. By contrast, as discussed above, associating physical meaning with transformation and eigenvector eigenvalue is more indirect, involving multiple steps of reasoning. We conjecture that other "new" symbolic forms involved in students' QM mathematical sensemaking may also display this kind of complexity or indirectness of physical interpretation.

\section{Primitive vs compound forms}

Transformation is primitive; it is not built out of other symbolic forms. By contrast, eigenvector eigenvalue is 
compound (nonprimitive), with a conceptual schema that is more complex than a single $p$-prim-like intuitive idea. We conjecture that, as compared to mathematical sensemaking in introductory physics, mathematical sensemaking in quantum mechanics will involve compound symbolic forms a higher percentage of the time.

This is analogous to the description that the resources framework $[38,53,54]$ gives for conceptual physics reasoning: the resources include basic everyday ideas such as $p$ prims [39], but also include more complex resources compiled from multiple elements [55].

\section{IMPLICATIONS AND CONCLUSIONS}

In this theory paper, using Fred and Gus as a touchstone, we introduced some constructs and conjectures about the nature of mathematical sense-making in quantum mechanics, how it might differ from "classical" mathematical sense-making in some ways but not others. We proposed that, in QM, metalevel reasoning about trustworthiness of and relations among, mathematical, conceptual, and intuitive reasoning might be essential to productive learning and problem solving. We also proposed that in QM mathematical sense-making, as in previously documented instances of mathematical sense-making in (mostly classical) physics, (i) parsing the conceptual meaning of mathematical expressions and equations can play a key role in mathematical sense-making, and (ii) Sherin's symbolic forms are useful for modeling the knowledge elements students activate when parsing those conceptual meanings. However, productive mathematical sense-making in QM requires new symbolic forms not documented in Sherin's original study, which took place in the context of classical, lower-division physics. We proposed two new forms, transformation and eigenvector eigenvalue, while noting that others likely exist as well. Again, while our work is based in the QM context, there are of course other areas of physics where these symbolic forms may be relevant to students' mathematical sense-making.

\section{A. Implications for instruction}

Because this is early theoretical work, not an empirical study, we need to be extremely cautious about recommending instructional moves. All we can do for now is point to instructional goals. Recently, PER has focused on the instructional need to improve (i) students' conceptual understanding of QM and (ii) students' facility with difficult mathematical formalism such as eigenvalue-eigenvector equations. To these important goals we add mathematical sense-making. Fred and Gus got stuck not because of inadequate conceptual understanding (they articulated that different PIAB "levels" should correspond to different constant energies), and not because of inadequate skill at solving eigenvalue-eigenvector equations (they did not need to actually obtain the energy eigenstates and eigenvalues to address the question at hand, whether $E$ is constant for the PIAB in the ground state). What they needed instead was a conceptual understanding, grounded perhaps in the eigenvector-eigenvalue symbolic form, of the meaning of the time-independent Schrödinger equation. So, Fred and Gus illustrate why we urge instructors to address students' mathematical sense-making in QM courses.

\section{B. Implications for research}

While Fred and Gus engaged in many aspects of mathematical sense-making, which has helped students make progress in other settings, it actually led them astray in this case. This raises a crucial focus for research: What domain-specific knowledge about the structures of QM equations is needed in order for students' mathematical sense-making to be productive, at least most of the time, toward getting correct answers? This question can guide future research on students' mathematical sense-making involving not only eigenvector-eigenvalue equations, but also other types of mathematical and physical structures relevant to quantum mechanics, including spin [56], inner products [57], and Hilbert space. While our description of the "new" symbolic forms relevant for quantum mechanics has been primarily theoretical, empirical observation of students using these symbolic forms will contribute to our understanding and help refine the theory.

\section{ACKNOWLEDGMENTS}

We thank Vesal Dini, Noah Finkelstein, Jessica Hoehn, Brandon James Johnson, and the UMD Physics Education Research Group for discussions that contributed to the analysis of the data. We thank the students who participated in our research. This work was supported by NSF-DUE Grants No. 13-23129 and No. 16-25797. Partial funding for open access provided by the UMD Libraries' Open Access Publishing Fund.

B. W. D. and A. E. contributed equally to this work.

\section{APPENDIX: TRANSCRIPT CONVENTIONS}

Parentheses are used to indicate gestures and actions, e.g., "(writing)", as well as pauses.

"[inaud.]" represents words that are inaudible or incomprehensible.

"[...]" means that some dialogue is skipped in the transcript included here. The transcript of the full episode is available in the Supplemental Material [58].

Slashes are used when one speaker talks over another, e.g., "Fred: So even if we divide this over, /Gus: Well.../ it would still be"

An em dash indicates when a speaker is cut off (e.g., "Gus: Oh, ok. Kind of the same as the previous one, which is-") or cuts themselves off (e.g., "Gus: 'Cause $V$, oryeah, $V$ will be the same here"). 
[1] I. D. Johnston, K. Crawford, and P. R. Fletcher, Student difficulties in learning quantum mechanics, Int. J. Sci. Educ. 20, 427 (1998).

[2] C. Singh, Student understanding of quantum mechanics, Am. J. Phys. 69, 885 (2001).

[3] C. Singh and E. Marshman, Review of student difficulties in upper-level quantum mechanics, Phys. Rev. ST Phys. Educ. Res. 11, 020117 (2015).

[4] E. Marshman and C. Singh, Framework for understanding the patterns of student difficulties in quantum mechanics, Phys. Rev. ST Phys. Educ. Res. 11, 020119 (2015).

[5] J. Petri and H. Niedderer, A learning pathway in highschool level quantum atomic physics, Int. J. Sci. Educ. 20, 1075 (1998).

[6] G. Ireson, The quantum understanding of pre-university physics students, Phys. Educ. 35, 15 (2000).

[7] K. Mannila, I. T. Koponen, and J. A. Niskanen, Building a picture of students' conceptions of wave-and particlelike properties of quantum entities, Eur. J. Phys. 23, 45 (2002).

[8] G. Kalkanis, P. Hadzidaki, and D. Stavrou, An instructional model for a radical conceptual change towards quantum mechanics concepts, Sci. Educ. 87, 257 (2003).

[9] K. S. Taber, Learning quanta: Barriers to stimulating transitions in student understanding of orbital ideas, Sci. Educ. 89, 94 (2005).

[10] B.W. Dreyfus, E. R. Sohr, A. Gupta, and A. Elby, "Classical-ish": Negotiating the boundary between classical and quantum particles, in Physics Education Research Conference Proceedings 2015, College Park, MD (AIP, New York, 2015), pp. 111-114.

[11] C. Baily and N. D. Finkelstein, Teaching and understanding of quantum interpretations in modern physics courses, Phys. Rev. ST Phys. Educ. Res. 6, 010101 (2010).

[12] C. Baily and N. D. Finkelstein, Teaching quantum interpretations: Revisiting the goals and practices of introductory quantum physics courses, Phys. Rev. ST Phys. Educ. Res. 11, 020124 (2015).

[13] E. Gire and E. Price, Structural features of algebraic quantum notations, Phys. Rev. ST Phys. Educ. Res. 11, 020109 (2015).

[14] P. J. Emigh, G. Passante, and P. S. Shaffer, Student understanding of time dependence in quantum mechanics, Phys. Rev. ST Phys. Educ. Res. 11, 020112 (2015).

[15] G. Zhu and C. Singh, Improving students' understanding of quantum measurement. I. Investigation of difficulties, Phys. Rev. ST Phys. Educ. Res. 8, 010117 (2012).

[16] G. Passante, P. J. Emigh, and P. S. Shaffer, Examining student ideas about energy measurements on quantum states across undergraduate and graduate levels, Phys. Rev. ST Phys. Educ. Res. 11, 020111 (2015).

[17] G. Passante, P. J. Emigh, and P. S. Shaffer, Student ability to distinguish between superposition states and mixed states in quantum mechanics, Phys. Rev. ST Phys. Educ. Res. 11, 020135 (2015).

[18] A. H. Schoenfeld, On Mathematics as Sense-Making: An Informal Attack on the Unfortunate Divorce of Formal, and Informal Mathematics, in Informal Reasoning, and Education, edited by J. F. Voss, D. N. Perkins, and J. W. Segal
(Lawrence Erlbaum Associates, Hillsdale, NJ, 1991), pp. 311-342.

[19] A. H. Schoenfeld, Learning to Think Mathematically: Problem Solving, Metacognition, and Sense Making in Mathematics, in Handbook for Research on Mathematics Teaching and Learning, edited by D. A. Grouws (Macmillan, New York, 1992), pp. 334-370.

[20] P. Heller, Teaching problem solving through cooperative grouping. Part 1: Group versus individual problem solving, Am. J. Phys. 60, 627 (1992).

[21] F. Reif, Millikan Lecture 1994: Understanding and teaching important scientific thought processes, Am. J. Phys. 63, 17 (1995).

[22] J. Tuminaro and E. F. Redish, Elements of a cognitive model of physics problem solving: Epistemic games, Phys. Rev. ST Phys. Educ. Res. 3, 020101 (2007).

[23] E. Kuo, M. M. Hull, A. Gupta, and A. Elby, How students blend conceptual and formal mathematical reasoning in solving physics problems: Conceptual and formal mathematical reasoning, Sci. Educ. 97, 32 (2013).

[24] M. M. Hull, E. Kuo, A. Gupta, and A. Elby, Problemsolving rubrics revisited: Attending to the blending of informal conceptual and formal mathematical reasoning, Phys. Rev. ST Phys. Educ. Res. 9, 010105 (2013).

[25] T. J. Bing and E. F. Redish, Epistemic complexity and the journeyman-expert transition, Phys. Rev. ST Phys. Educ. Res. 8, 010105 (2012).

[26] C. Singh, Student understanding of quantum mechanics at the beginning of graduate instruction, Am. J. Phys. 76, 277 (2008).

[27] B. R. Wilcox and S. J. Pollock, Upper-division student difficulties with the Dirac delta function, Phys. Rev. ST Phys. Educ. Res. 11, 010108 (2015).

[28] T. I. Smith, D. B. Mountcastle, and J. R. Thompson, Student understanding of the Boltzmann factor, Phys. Rev. ST Phys. Educ. Res. 11, 020123 (2015).

[29] B. L. Sherin, How students understand physics equations, Cognit. Instr. 19, 479 (2001).

[30] A. Gupta and A. Elby, Beyond epistemological deficits: Dynamic explanations of engineering students' difficulties with mathematical sense-making, Int. J. Sci. Educ. 33, 2463 (2011).

[31] M. Wertheimer, Productive Thinking (Harper, New York, 1945).

[32] G. Hatano and K. Inagaki, Two courses of expertise, in Child Development and Education in Japan, edited by H. W. Stevenson, H. Azuma, and K. Hakuta (W H Freeman Times Books Henry Holt \& Co, New York, 1986), pp. 262-272.

[33] A. Izsak, Students' coordination of knowledge when learning to model physical situations, Cognit. Instr. 22, 81 (2004).

[34] E. R. Sohr, B. W. Dreyfus, A. Gupta, and A. Elby, "Because math": Epistemological stance or defusing social tension in quantum mechanics?, in Physics Education Research Conference Proceedings 2015, College Park, MD (AIP, New York, 2015), pp. 319-322.

[35] Tutorials on thinking about quantum entities, https://www .physport.org/curricula/QuantumEntities/. 
[36] B. Jordan and A. Henderson, Interaction analysis: Foundations and practice, J. Learn. Sci. 4, 39 (1995).

[37] M. Eisenhart, Generalization from qualitative inquiry, in K. Ercikan and W.-M. Roth, editors, Generalizing from Educational Research: Beyond Qualitative and Quantitative Polarization (Routledge, New York, 2009).

[38] D. Hammer, Student resources for learning introductory physics, Am. J. Phys. 68, S52 (2000).

[39] A. A. diSessa, Toward an epistemology of physics, Cognit. Instr. 10, 105 (1993).

[40] D. Hammer and A. Elby, Tapping epistemological resources for learning physics, J. Learn. Sci. 12, 53 (2003).

[41] D. Hammer, Epistemological beliefs in introductory physics, Cognit. Instr. 12, 151 (1994).

[42] R. E. Scherr, H. G. Close, S. B. McKagan, and S. Vokos, Representing energy. I. Representing a substance ontology for energy, Phys. Rev. ST Phys. Educ. Res. 8, 020114 (2012).

[43] B. W. Dreyfus, B. D. Geller, J. Gouvea, V. Sawtelle, C. Turpen, and E. F. Redish, Ontological metaphors for negative energy in an interdisciplinary context, Phys. Rev. ST Phys. Educ. Res. 10, 020108 (2014).

[44] A. Johansson, S. Andersson, M. Salminen-Karlsson, and M. Elmgren, "Shut up and calculate": the available discursive positions in quantum physics courses, Cult. Stud. Sci. Educ. 1 (2016).

[45] B. W. Dreyfus, J. Hoehn, A. Elby, N. D. Finkelstein, and A. Gupta, Splits in students' attitudes toward classical and quantum physics (in preparation).

[46] B. A. Danielak, A. Gupta, and A. Elby, Marginalized identities of sense-makers: Reframing engineering student retention, J. Eng. Educ. 103, 8 (2014).

[47] L. Lising and A. Elby, The impact of epistemology on learning: A case study from introductory physics, Am. J. Phys. 73, 372 (2005).

[48] T. J. Bing and E. F. Redish, Analyzing problem solving using math in physics: Epistemological framing via warrants, Phys. Rev. ST Phys. Educ. Res. 5, 020108 (2009).

[49] A. Kaczynski and M. C. Wittmann, Student expectations in a group learning activity on harmonic motion, AIP Conf. Proc. 1513, 210 (2013).

[50] M. O. J. Thomas and S. Stewart, Eigenvalues and eigenvectors: embodied, symbolic and formal thinking, Math. Educ. Res. J. 23, 275 (2011).

[51] D. Z. Alaee, K. Kornick, E. C. Sayre, and S. V. Franklin, What Physicists Mean By The Equals Sign In Undergraduate Education (2017), http://meetings.aps.org/link/ BAPS.2017.APR.Y2.5.

[52] E. J. Knuth, A. C. Stephens, N. M. McNeil, and M. W. Alibali, Does understanding the equal sign matter? evidence from solving equations, J. Res. Math. Educ. 37, 297 (2006).

[53] A. Elby, Helping physics students learn how to learn, Am. J. Phys. 69, S54 (2001).

[54] E. F. Redish, A theoretical framework for physics education research: Modeling student thinking, in Proceedings of the International School of Physics, edited by E. F. Redish and M. Vicentini (IOS Press, Varenna, Italy, 2003), Vol. 156, pp. 1-64.

[55] E. Sayre and M. Wittmann, Plasticity of intermediate mechanics students' coordinate system choice, Phys. Rev. ST Phys. Educ. Res. 4, 020105 (2008).

[56] H. G. Close, E. W. Close, and D. Donnelly, Nesting in graphical representations in physics, in 2012 Physics Education Research Conference Proceedings, AIP Conf. Proc. 1513, 110 (2013).

[57] G. Passante, P. J. Emigh, T. Wan, and P. S. Shaffer, Investigating student understanding of perturbation theory and the inner products of functions, in Physics Education Research Conference Proceedings 2015, College Park, MD (AIP, New York, 2015), pp. 247-250.

[58] See Supplemental Material at http://link.aps.org/ supplemental/10.1103/PhysRevPhysEducRes.13.020141 for a transcript of the full episode. 